\title{
A STUDY ON FUZZY ROBUST REGRESSION AND ITS APPLICATION TO INSURANCE
}

\author{
Kamile Şanlı Kula ${ }^{1}$, Fatih Tank², Türkan Erbay Dalkılıç \\ ${ }^{1}$ Department of Mathematics, Ahi Evran University \\ 40200, Kırşehir / TURKEY, sanli2004@hotmail.com \\ ${ }^{2}$ Department of Statistics, Ankara University \\ 06100, Tandoğan, Ankara, TURKEY, tank@ankara.edu.tr \\ ${ }^{3}$ Department of Statistics and Computer Sciences, Karadeniz Technical University \\ 61080, Trabzon, TURKEY, tedalkilic@gmail.com
}

\begin{abstract}
In this study, a fuzzy robust regression method is proposed to construct a model that describes the relation between dependent and independent variables in insurance. Fuzzy robust regression suggested as an alternative to not only ordinary least squares but also classical robust regression. Fuzzy robust regression is finally investigated and discussed by an example with real data arose from a well-known insurance company in Turkey.
\end{abstract}

Key Words - robust regression, fuzzy regression, membership function, insurance

\section{INTRODUCTION}

Ordinary least squares (LS) method is one of the most well-known and the simplest type of estimation tool in statistics to determine the line of the best fit for a model. The LS method is specified by an equation with certain parameters to observed data and used in regression analysis and estimation with some assumptions, which can be easily found in the literature. Related assumptions of the LS method may be easily found in the literature. However, in the parameter estimation of the regression, the method has also some incompetence when the data has the outlier, and underlying distribution departs from normality. Therefore, diagnostic checks should be done on data before applying the LS method.

A statistical procedure is called robust if it is insensitive to the occurrence of gross errors in the data. Robust statistical procedures seek to provide methods even if there exists a single outlier. In such cases, robust methods are preferred to the LS method.

Fuzzy regression analysis is a fuzzy type of the classical regression analysis that is used in evaluating the functional relationship between the dependent and independent variables in a fuzzy environment[16]. The fuzzy regression model analysis is generally divided into two categories. The first of them is based on the Tanaka et al. (1982)'s linear programming approach, and the second one is based on the fuzzy least squares approach which is proposed by Diamond (1988) [16][18][25][26].

Tanaka et al.[22] proposed a study on linear regression with a fuzzy model then fuzzy regression analysis has been widely studied and applied in various areas. However, Tanaka's approach may give an incorrect interpretation of the fuzzy linear regression results when the data set consists at least an outlier. To handle the outlier 
problem, Hung and Yang (2006) proposed an omission approach for Tanaka's linear programming method. This approach had the capability to examine the behavior of value changes in the objective function of fuzzy regression models when observations are omitted. The procedure is to delete the first $i$-th observation and then the remaining observations are applied Tanaka's approach[12]. Nasrabadi et al. (2007) developed a new linear-programming-based approach for computations of fuzzy regression models [17].

In the context of fuzzy regression, observation which has a bigger residual value than the others is called as an outlier [12]. Fuzzy robust regression is robust for the estimation of fuzzy linear regression models, especially when outliers exist.

Rousseeuw et al.'s (1984) model considered a simple regression model. Furthermore, dependent and independent variables are represented as are non-fuzzy (crisp) numbers and estimations of parameters are non-fuzzy (crisp) number [19].

In this study, we focused on a multi-regression model by using fuzzy numbers when dependent and independent variables are triangular fuzzy numbers and estimation of parameters is a crisp number.

This paper has been divided into five sections. Section 2 describes parameter estimation in multiple linear regression. Section 3 deals with the concepts of robust regression. An algorithm for parameter estimation on fuzzy robust regression and a numerical study, based on real data, will be investigated by using the algorithm in Section 4. Finally, in the last section, discussion and conclusion are provided.

\section{MULTIPLE LINEAR REGRESSION}

Regression analysis is a useful statistical tool to model the functional relationship between dependent and independent variables under regularity conditions. Multiple linear regression generalizes the simple linear regression model by allowing for many terms in a mean function rather than just one intercept and one slope [24].

In almost all cases, the outcome of a related variable is not determined entirely by the outcome of a single factor. Many independent factors typically have an effect on the dependent variable. For instance, total claim amount payments of car accidents may be affected by time period of the year, vehicle type or/and number of accidents. Thus, an extension of the classical linear model might assume that the dependent variable is determined by a linear combination of the independent variables. For any observation $i$ :

$$
Y_{i}=\beta_{0}+\beta_{1} X_{i 1}+\beta_{2} X_{i 2}+\ldots+\beta_{j} X_{i j}+\varepsilon_{i} \text {, for } j=1,2, \ldots, p ; i=1,2, \ldots, n \text {. }
$$

Generally model, which is given in (1), is denoted in matrix form as:

$$
\mathbf{Y}=\mathbf{X} \beta+\varepsilon \text {. }
$$

where, $\mathbf{Y}$ is $n \times 1$ matrix of $n$ observations, $\beta$ is $(k+1) \times 1$ matrix of the beta coefficients, $\mathbf{X}$ is $n \times(k+1)$ matrix containing $n$ observations for $k$ independent variables and $\varepsilon$ is $n \times 1$ matrix of error terms. Variables must be linearly independent for fitting a linear regression model. Otherwise the estimations of the coefficients cannot be determined. We assume that the independent variable is non-random for repeated samples, and the error terms are random with a zero mean conditional on the explanatory variables and $E\left(\varepsilon^{\prime} \varepsilon\right)=\sigma^{2}$. The error term essentially describes that the 
error in the predicted value, given the independent variables [1]. Solving for $\beta$ coefficients, the goal is to minimize the sum of squares error, which can be expressed by

$$
\varepsilon^{\prime} \varepsilon=\sum_{i=1}^{n} \varepsilon_{i}^{2}
$$

This can also be expressed as

$$
(\mathbf{Y}-\mathbf{X} \beta)^{\prime}(\mathbf{Y}-\mathbf{X} \beta) \text {. }
$$

By taking the first partial derivative with respect to $\widehat{\beta}$ and setting it to zero gives the solution as

$$
\widehat{\beta}=\left[\mathbf{X}^{\prime} \mathbf{X}\right]^{-1} \mathbf{X}^{\prime} \mathbf{Y}
$$

In here, $\widehat{\beta}$ is called as estimation of multiple linear regression coefficients. The main advantage of multiple linear regression is that it allows the use of more than one variable to explain the variation of the dependent variable $Y$. Remember that more independent variables may not always give better estimation of the dependent variable $Y[8]$.

\section{ROBUST REGRESSION}

LS estimator is very sensitive to outliers and to deviation from normality assumptions. Therefore, estimation results are directly affected by each observation and the data should be analyzed in detail. This is such an important issue that sometimes even a single observation can change the value of the parameter estimates dramatically, and omitting this observation from the data may lead to totally different results. Outliers always have huge impact on model fitting, especially in regression analysis. They decrease the efficiency of the estimator. Now, we discuss widely used M estimation methods of Huber, Hampel, Andrews and Tukey.

The most common general method of robust regression is $\mathrm{M}$ estimations, introduced by Huber(1964) [10][16]. This class of estimators can be regarded as a generalization of maximum-likelihood estimation, hence the term ' $M$ ', comes from "generalized maximum likelihood" estimation [7]. The general M estimator minimizes the objective function

$$
\sum_{i=1}^{n} \rho\left[\left(y_{i}-\sum_{j=1}^{p} x_{i j} \widehat{\beta}_{j}\right) / d\right]
$$

where the function $\rho$ gives the contribution of each residual to the objective function. By taking the first degree partial derivative of the sum in equation (4) with respect to each $\widehat{\beta}_{j}$ and setting it to zero, it may be found that regression coefficients of $p$ equations:

$$
\sum_{i=1}^{n} x_{i j} \Psi\left[\left(y_{i}-\sum_{j=1}^{p} x_{i j} \widehat{\beta}_{j}\right) / d\right]=0, j=1,2, \ldots, p
$$


where $\Psi(z)=\rho^{\prime}(z)$ be the derivative of $\rho$. The standardized residuals may be defined as $z=r_{i} / d$ where $r_{i}=y_{i}-\sum_{j=1}^{p} x_{i j} \widehat{\beta}_{j}$ [14]. In here, $d$ is a robust estimate of scale [14]. Under normality, the expected value of $d$ is the standart error of estimate in population [13]. When data contain outliers, standart deviations are not a good measures of variability. Hence, other robust measures of variability are required. One robust measure of variability $(d)$ is defined as;

$$
d=\text { median } \mid r_{i}-\text { median }\left(r_{i}\right) \mid / 0.6745
$$

0.6745 is used as denominator in equation (6) because then $d \approx \sigma$ when $n$ is large and the sample actually arises from a normal distribution. Usually the sample standart deviation $s$ is not used as a $d$ value since it is influenced too much by outliers and thus is not robust [13]. The numerator of $d$ in equation (6) is called as the median of the absolute deviations (MAD) [11].

Huber's $\rho$ is defined as

$$
\rho(z)=\left\{\begin{array}{cl}
\frac{z^{2}}{2} & ,|z| \leq k \\
k|z|-\frac{k^{2}}{2} & ,|z|>k
\end{array} .\right.
$$

The cutoff point is referred to in robust statistics as a tuning constant. When the normal error structure holds (e.g., when least square estimates typically are considered best), the use of the Huber method would result in some loss of efficiency. The percentage of loss in efficiency represent the Premium to be paid for the guard against un-reasonable least square estimates in nonnormal cases. If the Premium is set at 5\%, the tuning constant $k$ is typically set at 1.5 [13]. The tuning constant is generally picked to give reasonably high efficiency in the normal case; in particular, for the Huber produce percent efficiency when the errors are normal, and still offer protection against outliers [7]. Huber's weight function is defined by $W(z)=\Psi(z) / z$ such as

$$
W(z)= \begin{cases}1 & ,|z| \leq k \\ \frac{k}{z} & ,|z|>k\end{cases}
$$

$[5][7][13][14]$.

The Hampel's $\rho$ and $W$ functions are defined as: 


$$
\rho(z)=\left\{\begin{array}{cl}
\frac{z^{2}}{2} & , 0<|z| \leq a \\
a|z|-\frac{a^{2}}{2} & , a<|z| \leq b \\
-\frac{a(c-z)^{2}}{2(c-b)}+\frac{a(b+c-a)}{2} & , b<|z| \leq c \\
\frac{a(b+c-a)}{2} & , c<|z| \\
\left.\frac{1}{z}\right)=\left\{\begin{array}{cl}
\frac{a}{z} \operatorname{sgn}(z) & , a<|z| \leq b \\
\frac{a}{z}\left(\frac{c-|z|}{c-b}\right) \operatorname{sgn}(z) & , b<|z| \leq c \\
0 & , c<|z|
\end{array}\right.
\end{array}\right.
$$

respectively. In here, $a=1.7, b=3.4$ and $c=8.5$ are called cutoff point of the Hampelestimator. Reasonably good choices for the constant are $a=1.7, b=3.4$ and $c=8.5$ [5][9][13][14].

The Andrew's $\rho$ and $W$ functions are defined as:

$$
\rho(z)=\left\{\begin{array}{cl}
k^{2}\left(1-\cos \frac{|z|}{k}\right) & ,|z| \leq k \pi \\
2 k^{2} & ,|z|>k \pi
\end{array} \quad W(z)=\left\{\begin{array}{cc}
\frac{\sin (z / k)}{z} & ,|z| \leq k \pi \\
0 & ,|z|>k \pi
\end{array}\right.\right.
$$

respectively. In here, $k$ is called cutoff point of the Andrews-estimator. Actually if the scale is known, $k=1.339$ requires a premium of $5 \%$ otherwise $k=1.5$ or $k=2.1$ [5][9] [13][14].

The Tukey's bisquare (or biweight estimator) $\rho$ and $W$ functions are defined as:

$$
\rho(z)=\left\{\begin{array}{cl}
\frac{1}{6}\left(1-\left(1-\left(\frac{z}{k}\right)^{2}\right)^{3}\right) & ,|z| \leq k \\
\frac{1}{6} & ,|z|>k
\end{array} \quad W(z)=\left\{\begin{array}{cl}
\left(1-(z / k)^{2}\right)^{2} & ,|z| \leq k \\
0 & ,|z|>k
\end{array}\right.\right.
$$

respectively. In here, $k$ is called cutoff point of the Tukey-estimator. If scale is known, $k=4.685$ implies a premium of $5 \%$ otherwise $k=5.0$ or $k=6.0$ [5][7][9][13][14].

A robust procedure tries to accommodate the majority of the data. Outliers consequently possess large residuals from the robust fit. So, in addition to insensitivity to outliers, a robust regression estimator makes the detection of the outliers as an easy job. Of course, the residual from LS cannot be used for this purpose, because the outliers may possess very small LS residual as the LS fit is pulled too much in the direction of these deviating points [20]. 


\section{AN ALGORITHM FOR PARAMETER ESTIMATIONS BY THE FUZZY ROBUST REGRESSION}

LS estimates are known to be the best when data have normal distributed error terms. Observation may be called an outlier when it has the largest residual in the context of fuzzy regression [12]. The classical LS method is influenced by the outliers and outliers may distort the estimates. Accordingly, robust methods have been created to modify LS methods so that the outliers have much less influence on the final estimates. The fuzzy robust regression is robust for the estimation of fuzzy linear regression models, when outliers are exist in the given data set. In case of outliers in a data set, the estimation of regression parameters has been studied by many authors and robust methods have been defined. Recently, much research has studied fuzzy estimation [15].

In this study, we consider a multi-regression model by using fuzzy numbers when $X$ and $Y$ are triangular fuzzy numbers, estimation of parameters are crisp numbers. In the model estimation, heuristics are not allowed. After the multi-regression model is obtained, it is determined whether outliers exist in the given data set, and the weight matrix is defined by the membership function of the residuals. The weighted fuzzy least square is built by using the weight matrix. Thus, a model estimation not influenced by outliers is obtained. The fuzzy robust regression method may be able to detect outliers automatically by giving each one a membership that is zero or very small as compared with other memberships.

Let $X_{1}, X_{2}, \ldots, X_{p}$ be independent variables. Triangular fuzzy numbers are defined as $[X=(X, \underline{\xi}, \bar{\xi})]$ where $x$ is the modal value of $X, \underline{\xi}$ is left spreads and $\bar{\xi}$ is right spreads. Let $Y$ be a dependent variable. Triangular fuzzy numbers are defined as $[Y=(y, \underline{\eta}, \bar{\eta})]$ where $y$ is the modal value of $Y, \underline{\eta}$ is left spreads and $\bar{\eta}$ is right spreads. According to the information given above, dependent and independent variables are triangular fuzzy numbers, the steps of the fuzzy robust regression method algorithm are as follows:

Step 1: When $X_{i}=\left(x_{i}, \underline{\xi_{i}}, \bar{\xi}_{i}\right)$ and $Y_{i}=\left(y_{i}, \underline{\eta_{i}}, \overline{\eta_{i}}\right), i=1,2, \ldots, n$, triangular fuzzy numbers, fuzzy regression model is defined by:

$$
Y=a+b X
$$

where $a, b$ are crisp numbers. When parameters are crisp, the fuzzy least squares optimization problem is defined as:

$$
\min r(a, b)=\sum d\left(a+b X_{i}, Y_{i}\right)^{2},
$$

In equation (7), $d\left(a+b X_{i}, Y_{i}\right)$ is

$$
\begin{aligned}
d\left(a+b X_{i}, Y_{i}\right)^{2} & =\left[a+b x_{i}-y_{i}-\left(b \underline{\xi_{i}}-\underline{\eta_{i}}\right)\right]^{2}+\left[a+b x_{i}-y_{i}-\left(b \bar{\xi}_{i}-\bar{\eta}_{i}\right)\right]^{2} \\
& +\left(a+b x_{i}-y_{i}\right)^{2}
\end{aligned}
$$

[6]. In this study, the fuzzy least squares model is given in equation (7) by generalized multi-regression model. In this case, the optimization problem is defined as: 


$$
\min r\left(a, b_{1}, b_{2}, \ldots, b_{p}\right)=\sum d\left(a+b_{1} X_{i 1}+b_{2} X_{i 2}, \ldots, b_{p} X_{i p}, Y_{i}\right)^{2} .
$$

The parameters are estimated by the minimizing equation (8). Estimation of parameter is found as equation (9) [21]. When $X=(x, \underline{\xi}, \bar{\xi})$ and $Y=(y, \underline{\eta}, \bar{\eta})$ are a triangular fuzzy numbers, initial estimation of parameter is found as

$$
\widehat{\beta}=\left(X^{\prime} X+A^{\prime} A+B^{\prime} B\right)^{-1}\left(X^{\prime} Y+A^{\prime} C+B^{\prime} D\right)
$$

where

$$
\begin{aligned}
X & =\left[\begin{array}{cccc}
1 & x_{11} & \ldots & x_{1 p} \\
1 & x_{21} & \ldots & x_{2 p} \\
\vdots & \vdots & \ddots & \vdots \\
1 & x_{n 1} & \ldots & x_{n p}
\end{array}\right], Y=\left[\begin{array}{c}
y_{1} \\
y_{2} \\
\vdots \\
y_{n}
\end{array}\right], C=\left[\begin{array}{c}
y_{1}-\underline{\eta}_{1} \\
y_{2}-\underline{\eta}_{2} \\
\vdots \\
y_{n}-\underline{\eta}_{n}
\end{array}\right], D=\left[\begin{array}{c}
y_{1}+\bar{\eta}_{1} \\
y_{2}+\bar{\eta}_{2} \\
\vdots \\
y_{n}+\bar{\eta}_{n}
\end{array}\right] \\
A & =\left[\begin{array}{cccc}
1 & x_{11}-\underline{\xi}_{11} & \ldots & x_{1 p}-\underline{\xi}_{1 p} \\
1 & x_{21}-\underline{\xi}_{21} & \ldots & x_{2 p}-\underline{\xi}_{2 p} \\
\vdots & \vdots & \ddots & \vdots \\
1 & x_{n 1}-\underline{\xi}_{n 1} & \ldots & x_{n p}-\underline{\xi}_{n p}
\end{array}\right], B=\left[\begin{array}{cccc}
1 & x_{11}+\bar{\xi}_{11} & \ldots & x_{1 p}+\bar{\xi}_{1 p} \\
1 & x_{21}+\bar{\xi}_{21} & \ldots & x_{1 p}+\bar{\xi}_{1 p} \\
\vdots & \vdots & \ddots & \vdots \\
1 & x_{n 1}+\bar{\xi}_{n 1} & \ldots & x_{1 p}+\bar{\xi}_{1 p}
\end{array}\right]
\end{aligned}
$$

provided that the $\left(X^{\prime} X\right)^{-1}$ is exist.

Step 2: $\hat{y}_{i}$ are estimated and the residuals $r_{i}$ are calculated.

Step 3: Median is determined with respect to the absolute residual values, and distances are calculated by

$$
D_{i}=\| \operatorname{abs}\left(r_{i}\right)-\text { median }\left(\operatorname{abs}\left(r_{i}\right)\right) \|, i=1,2, \ldots, n
$$

where $\|$.$\| is the Euclidean distance[21].$

Step 4: The membership function is shown in Figure 1 and defined in as follows:

$$
\mu(r)=\left\{\begin{array}{cc}
1 & ,|r| \leq a \\
\frac{b-|r|}{b-a} & , a<|r|<b \\
0 & , \text { elsewhere }
\end{array}\right.
$$

where $\quad a=$ median $\left(D_{i}\right) \quad$ and $\quad b=\max \left(D_{i}\right)+d . \quad$ In here

$d=$ median $\mid r_{i}-$ median $\left(r_{i}\right) \mid / 0.6745$ 


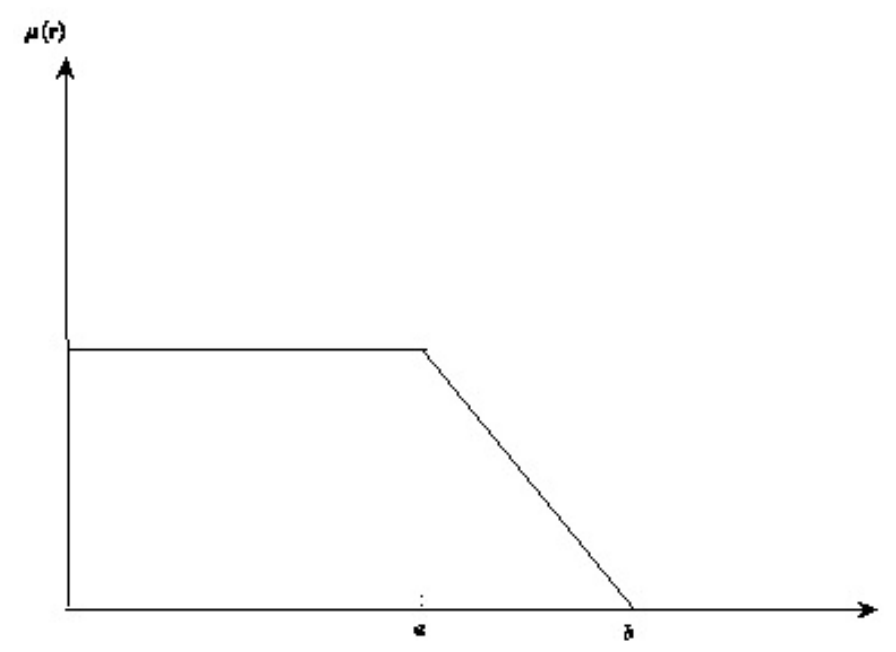

Figure1. Picture of the membership function.

Step 5: Chang and Lee (1996) proposed a generalized fuzzy weighted least squares regression. The model based interactive decision maker and used non-fuzzy inputoutput data[2]. Weighted function is consists the degree of membership and the weighted fuzzy least square is built by using the weight matrix [21]. The membership function defined by equation (10), the membership values are determined and the weight matrix is constituted. The weight matrix is a diagonal matrix, where diagonal elements consist of the degree of membership. The weighted fuzzy least squares estimations are found by

$$
\widehat{\beta}=\left(X^{\prime} W X+A^{\prime} W A+B^{\prime} W B\right)^{-1}\left(X^{\prime} W Y+A^{\prime} W C+B^{\prime} W D\right)
$$

provided that the $\left(X^{\prime} X\right)^{-1}$ is exist and $W$ is non-zero matrix where $W$ is the weight matrix such that $W=\operatorname{diag}\left(\mu_{1}, \mu_{2}, \ldots, \mu_{n}\right)$.

Step 6: If $\left|\hat{\beta}^{k+1}-\hat{\beta}^{k}\right|<\varepsilon$ then stop. Otherwise proceed to Step 2. Where $\hat{\beta}$ is the estimation of regression parameters, $k$ denote the iteration number and $\varepsilon>0$ is a very small number [21].

\section{NUMERICAL EXAMPLE}

In our example the data are collected from a well-known insurance company. $X_{1}, X_{2}$ and $Y$ represent number of months, claim numbers in related month and payments in related months respectively. In this study the structure of the fuzziness is apart from the study of Dalkilic et al. (2009).

In Diamond 1988, while crisp numbers were fuzzified, left and right spread are calculated as approximately of centers $10 \%, 15 \%$ or $20 \%$ [6]. While crisp numbers were fuzzified for symmetric triangular fuzzy number, all spreads were calculated as 1 in Chang (2001) and asymmetric triangular fuzzy numbers were calculated by assigning left and right spreads halfwidths to the fuzzy data [3]. In Xu and Li (2001), the values of spread were assumed by the author[23]. 
In here, the independent variable values are fuzzified, analysis of the fuzzy robust regression (FRR) method takes the values of independent variables; center, left spread and right spread are defined as $x_{i}, \underline{\xi}_{i}=x_{i} / 8$ and $\bar{\xi}_{i}=x_{i} / 7$, respectively. The dependent variable value is fuzzified, FRR method takes the values of dependent variables; center, left spread and right spread are defined as $y_{i}, \underline{\eta}_{i}=y_{i} / 8$ and $\bar{\eta}_{i}=y_{i} / 7$, respectively.

Table 1. Data set

\begin{tabular}{|c|c|c|c|c|c|c|c|c|c|c|c|c|}
\hline$X_{1}$ & 1 & 2 & 3 & 4 & 5 & 6 & 7 & 8 & 9 & 10 & 11 & 12 \\
\hline$X_{2}$ & 1270 & 2630 & 3653 & 3045 & 3232 & 3681 & 3169 & 3448 & 3163 & 3096 & 3765 & 4481 \\
\hline$Y * 10^{4}$ & 125 & 387 & 589 & 591 & 609 & 654 & 631 & 545 & 583 & 606 & 753 & 898 \\
\hline
\end{tabular}

Analysis was performed using MATLAB code, which has written by the authors, for the LS method, M estimator and FRR. The results obtained by using data set, which has given in Table 1 . The results of the residual analysis indicate that the eighth observation is an outlier. Because, standardized residuals for this observation is greater than 2 .

Table 2. Estimation of Regression Parameters

\begin{tabular}{|c|c|c|c|c|c|c|}
\hline Estimations\Method & LS & Huber & Hampel & Tukey & Andrews & FRR \\
\hline$\widehat{\beta}_{0}$ & -118.4504 & -123.0439 & -121.3489 & -130.7774 & -120.6027 & -103.9568 \\
\hline$\widehat{\beta}_{1}$ & 12.2628 & 14.1149 & 13.8047 & 16.5405 & 13.2381 & 15.8823 \\
\hline$\widehat{\beta}_{2}$ & 0.1925 & 0.1900 & 0.1908 & 0.1847 & 0.1916 & 0.1804 \\
\hline
\end{tabular}

The parameter estimations of regression models are given in Table 2 for the different methods. As Table 2 shows, parameter estimations are the same in sign and nearly the same in magnitude as those obtained with robust methods, since the weight matrix is obtained via the membership function. Each observation is included in estimation of the regression model depending on the degree of membership. Thus possible negative effects of the outlier on the model may be minimized. FRR is not influenced by outliers, and results of the method are also beter.

The residuals and weights are obtained by LS, the M and the FRR methods and shown in Table 3 and Table 4, respectively. The weight matrix is obtained via the membership function. Each observation is included in estimation of the regression model depending on the degree of membership. Thus possible negative effects of the outlier on the model may be minimized. Our method is not influenced by outliers, and results of the method are also better than the others.

Parameter estimation of the regression model is obtained where $X$ and $Y$ are a triangular fuzzy numbers. In this case, it is seen that the model has less influenced by outliers than LS method. Therefore this approach allows to determine total claim amounts in the related month as an alternative to the model suggested by Rousseeuw et. al.

As mentioned in Section 3, contrary to rest of the observation's residuals, the 
eighth observation was an outlier due to it's large residual. So, it is seen that the residual, which are obtained via fuzzy robust regression method, has very big values such as $(-100.0739,82.1510)$ for outlier. Contribution of the other observations to the regression model is more significant and residuals are small for the observations except for outlier.

Table 3.Residuals for the LS, the M Method and the FRR Method

\begin{tabular}{|c|r|r|r|r|r|r|}
\hline Obs. \# $\backslash$ Method & \multicolumn{1}{|c|}{ LS } & \multicolumn{1}{c|}{ Huber } & \multicolumn{1}{c|}{ Hampel } & \multicolumn{1}{c|}{ Tukey } & \multicolumn{1}{c|}{ Andrews } & \multicolumn{1}{c|}{ FRR } \\
\hline 1 & -13.2563 & -7.3226 & -9.7292 & 4.7034 & -11.0125 & -16.0162 \\
\hline 2 & -25.2858 & -17.7856 & -20.9763 & -0.9911 & -22.8748 & -15.2240 \\
\hline 3 & -32.4510 & -24.2315 & -27.9353 & -4.4511 & -30.1560 & -13.6415 \\
\hline 4 & 74.3113 & 79.1504 & 76.2446 & 93.2890 & 75.1203 & 82.1510 \\
\hline 5 & 44.0555 & 47.5126 & 44.7657 & 60.2149 & 44.0464 & 50.5365 \\
\hline 6 & -9.6288 & -6.8952 & -9.6938 & 5.7567 & -10.2360 & -1.3393 \\
\hline 7 & 53.6559 & 53.2504 & 51.1726 & 60.7683 & 51.6433 & 52.1362 \\
\hline 8 & -98.3076 & -99.8639 & -101.8568 & -93.2957 & -101.0611 & -100.0739 \\
\hline 9 & -17.7148 & -21.8397 & -23.2942 & -19.2046 & -21.6830 & -26.5461 \\
\hline 10 & 5.9182 & -0.2271 & -1.3186 & -0.3721 & 0.9185 & -7.3426 \\
\hline 11 & 11.8893 & 5.5735 & 4.2534 & 6.5418 & 6.4763 & 3.0966 \\
\hline 12 & 6.8141 & 0.4459 & -1.1405 & 2.7762 & 1.0272 & 3.0577 \\
\hline Sum of Sq. Error & 22503.91 & 22843.58 & 22783.41 & 25221.06 & 22636.21 & 23489.41 \\
\hline
\end{tabular}

Sum of squares error is given in Table 3 for LS, M and the FRR methods. However, sum of squares error were greater for $M$ method than the other methods due to the residual value of outlier in robust method. It is also seen that the sum of squares error of FRR's method is close to M method's. In Table 3, dependent and independent variables, values are the crisp number in LS and M estimations, as well as fuzzy numbers in FRR. Estimation of regression paremeters is crisp number in the all method.

The weights of the eight observation are " 0.3619 ", " 0.3525 ", " 0 ", " 0.2490 " and "0.0124" for the methods of Huber, Hampel, Tukey, Andrew and FRR respectively. Weights that are found in the results from the FRR method are the degrees of membership of each observation. These memberships show effects of observations to the model. Also, as in Table 4, outliers influence the model by very small degree of membership, the degrees of membership of the other observation values are 1 or close to 1 , and the effects of those on the estimation of the regression model are important. Standardized residual of fourth observation is close to 2 . Degree of membership of this observation is small. Thus, contribution of the observation to the model is small and the degree of membership of the observation value is 0.2146 .

Table 4.The weights for the LS, the M method and the FRR method

\begin{tabular}{|c|c|c|c|c|c|c|}
\hline Obs. \# \Method & LS & Huber & Hampel & Tukey & Andrews & FRR \\
\hline 1 & 1.0000 & 1.0000 & 1.0000 & 0.9808 & 0.4730 & 0.9608 \\
\hline 2 & 1.0000 & 1.0000 & 1.0000 & 0.9991 & 0.4625 & 0.9698 \\
\hline 3 & 1.0000 & 1.0000 & 1.0000 & 0.9828 & 0.4526 & 0.9876 \\
\hline 4 & 1.0000 & 0.4566 & 0.5497 & 0.0000 & 0.3407 & 0.2146 \\
\hline 5 & 1.0000 & 0.7607 & 0.9362 & 0.0000 & 0.4267 & 0.5713 \\
\hline 6 & 1.0000 & 1.0000 & 1.0000 & 0.9713 & 0.4734 & 1.0000 \\
\hline 7 & 1.0000 & 0.6787 & 0.8190 & 0.0000 & 0.4089 & 0.5533 \\
\hline
\end{tabular}




\begin{tabular}{|c|l|l|l|l|l|l|}
\hline 8 & 1.0000 & 0.3619 & 0.3525 & 0.0000 & 0.2490 & 0.0124 \\
\hline 9 & 1.0000 & 1.0000 & 1.0000 & 0.7043 & 0.4639 & 0.8420 \\
\hline 10 & 1.0000 & 1.0000 & 1.0000 & 0.9999 & 0.4762 & 1.0000 \\
\hline 11 & 1.0000 & 1.0000 & 1.0000 & 0.9630 & 0.4751 & 1.0000 \\
\hline 12 & 1.0000 & 1.0000 & 1.0000 & 0.9933 & 0.4762 & 1.0000 \\
\hline
\end{tabular}

\section{CONCLUSION}

In this study, a fuzzy robust regression method has been suggested to construct a model to describe the relation between dependent and independent variables, as an alternative to ordinary least squares and classical robust regression method in insurance. When Table 2 is examined, it is seen that estimations of regression parameters obtained via the fuzzy robust regression method are the same sign and nearly the same magnitude as those obtained with robust methods. Therefore, it can be said that the estimation of regression model is suitable excluding outlier.

Four sub-model based on fuzzy infreence rules was taken by In Dalkilic et al (2009) [4]. In their study, each set of observations in the Neural Networks approach with the fuzzy rules for the membership degrees are included in the model. Thus, contribution of the outlier value to the models is limited by membership degrees and possible negative effects to model may be minimized. Apparently, from Dalkilic et al. (2009), in this study, the weight matrix is obtained via the membership function. Each observation is included in estimation of the regression model depending on the degree of membership. Thus possible negative effects of the outlier on the model are minimized.

\section{ACKNOWLEDGMENTS}

First and foremost the authors offer their sincerest gratitude to their Ph.D. supervisors, Prof. Dr. Aysen Apaydin (Ankara University, Turkey) and Prof. Dr. Omer L. Gebizlioglu (Ankara University, Turkey), who have continuously provided support for them throughout their studies and academic life. The authors also would like to thank an anonymous referee for making useful suggestions to improve the manuscript.

\section{REFERENCES}

1. W.D. Berry, S. Feldman, Multiple Regression in Practice, Sage University Papers Series, Quantitative Applications in the Social Sciences, No. 07-050, 1985.

2. P.T. Chang, and E.S. Lee, A generalized fuzzy weighted least-squares regression, Fuzzy Sets and Systems 82: 289-298, 1996.

3. Y.H.O. Chang, Hybrid fuzzy least-squares regression analysis and its reliability measures. Fuzzy Sets and Systems 119: 225-246, 2001.

4. T.E. Dalkilic, F. Tank, and K.S. Kula, Neural networks approach for determining total claim amounts in insurance, Insurance: Mathematics and Economics 45: 236-241, 2009.

5. R.N. Dave, and R. Krishnapuram, Robust clustering methods: A unified view, IEEE Transactions on Fuzzy Systems 5: 270-293, 1997. 
6. P. Diamond, Fuzzy least squares, Information Science 46: 141-157, 1988.

7. J. Fox, An R and S-PLUS Companion to Applied Regression, Sage Publications, 2002.

8. D. N. Gujarati, Essentials of Econometrics, St. Louis, MO: McGraw-Hill, Inc., 1992.

9. F.R., Hampel, E.M., Ronchetti, Rousseeuw, P.J. and Stahel, W.A, Robust statistics, John Willey \& Sons, New-York, 1986.

10. P.J. Huber, Robust Estimation of a Location Parameter, Annals of Mathematical Statistics 35: 73-101, 1964.

11. P.J. Huber, Robust Statistics, John Willey \& Son, New York (1981).

12. W. L. Hung and M. S. Yang, An omission approach for detecting outliers in fuzzy regression models, Fuzzy Sets and Systems 157: 3109-3122 (2006).

13. H. Huynh, A comparison of four approaches to robust regression, Psychological Bulletin 92: 505-512 (1982).

14. R.V. Hogg, Statistical robustness: one view of its use in applications today, The American Statistican 33: 108-115 (1979).

15. K.S., Kula, A. Apaydin, Fuzzy Robust Regression Analaysis Based on the Ranking of Fuzzy Sets, Int. J. Uncertainty, Fuzziness and Knowledge-Based Systems (IJUFKS) 16: 663-681 (2008).

16. M.M. Nasrabadi and E. Nasrabadi, A Mathematical-programming approach to fuzzy linear regression analysis, Applied Mathematics and Computation 163: 977-989 (2004).

17. E. Nasrabadi, S. M. Hashemi and M. Ghatee, An LP approach to outliers detection in fuzzy regression analysis, Int. J. Uncertainty, Fuzziness and Knowledge-Based Systems (IJUFKS) 15: 441-456 (2007).

18. D.T. Redden and W.H. Woddall, Futher examination of fuzzy linear regression, Fuzzy Sets and Systems 79: 203-211 (1996).

19. P. Rousseeuw, B. Daniels, A.Leroy, Applying robust regression to insurance, Insurance: Mathematics and Economics 3: 67-72 (1984).

20. P.J. Rousseuw, and A.M. Leroy, Robust regression and outlier detection, John Wiley \& Sons New York (1997).

21. K. Sanli, A. Apaydin, The fuzzy robust regression analysis, the case of fuzzy data set has outlier, G.U.Journal of Science 17: 71-84 (2004).

22. H. Tanaka, S. Uegima and K. Asai, Linear regression analysis with fuzzy model, IEEE Trans. Systems Man Cybernet 12: 903-907 (1982).

23. R. Xu, and C. Li, Multidimensional least-squares fitting with a fuzzy model, Fuzzy Sets and Systems 119: 215-223 (2001).

24. S. Weisberg, Applied Linear Regression (Third Edition), John Willey \& Son, Hoboken, New Jersey (2005).

25. M. S. Yang and C. H. Ko, On cluster-wise fuzzy regression analysis, IEEE Transaction on Systems, Man and Cybernetics Part B: Cybernetics 27: 1-13 (1997).

26. M.S. Yang, and T.S. Lin, Fuzzy least-squares linear regression analysis for fuzzy input-output data, Fuzzy Sets and Systems 126: 389-399 (2002). 